\title{
Improved Detectability with a Polymer-based Trapping Device in Rapid HPLC Analysis for Ultra-low Levels of Bisphenol A (BPA) in Environmental Samples
}

\author{
Yoshiyuki Watabe,* Takuya Kondo,** Hiroe ImaI,** Masatoshi Morita,** Nobuo TanaKa,* \\ Jun HaGiNaKa, $* * *$ and Ken Hosoya $* \dagger$ \\ *Department of Polymer Science \& Engineering, Kyoto Institute of Technology, Matsugasaki, \\ Sakyo-ku, Kyoto 606-8585, Japan \\ **Endocrine Disrupters Research Laboratory, National Institute for Environmental Studies, \\ 16-2 Onogawa, Tsukuba, Ibaraki 305-8506, Japan \\ ***Faculty of Pharmaceutical Sciences, Mukogawa Women's University, \\ 11-68 Koshien Kyuban-cho, Nishinomiya, Hyogo 663-8179, Japan
}

\begin{abstract}
A new high-performance liquid chromatography (HPLC) method has been developed to detect ultra-low concentrations of bisphenol-A (BPA) (below $1 \mathrm{ng} / \mathrm{L}$ (ppt)) using column switching electrochemical detection (ECD). The results were superior to those obtained from manual pretreatment procedure with membrane stationary phase. BPA is inherently ubiquitous in the environment, including tools and solvents used for its analysis; to obtain meaningful results, therefore, the concentration of the overall BPA contamination must be below the detection limit for BPA using the analytical system. Therefore, purified water for preparing the standard BPA solution must be filtered with a hydrophobic membrane to suppress BPA background levels of contamination. In addition, we investigated methods for effectively preserving environmental water containing BPA. The addition of a small amount of ethylenediaminetetraacetic acid (EDTA) provided good recovery even after overnight storage. By employing these precautionary measures and procedures to reduce BPA contamination from the analytical procedure, we could accurately determine $1-10 \mathrm{ppt}$ of BPA in environmental water samples using a column switching HPLC system.
\end{abstract}

(Received September 12, 2003; Accepted October 31, 2003)

\section{Introduction}

Bisphenol-A (BPA, Fig. 1-A) is widely used as raw material of not only epoxy and polycarbonate resins but also internal coating of cans for beverages, ${ }^{1}$ dental bonding, ${ }^{2}$ and so on. BPA is emitted into the environment during our daily life as well as during industrial production. Nowadays the use of BPA is being limited but it still often appears in environmental water and has been brought to public attention as an endocrine disrupter.

It has recently been proposed that trace amounts of BPA show estrogenic activity even at concentrations less than as low as 1 $\mathrm{ng} / \mathrm{L}$ (ppt) level. ${ }^{3-5}$ The GCMS method is commonly used for BPA determination. However, the drawback to this method is that pretreatment of the samples requires approximately one day to be completed through complicated procedures. On the other hand, the HPLC method only requires simple sample pretreatment followed by immediate analysis. BPA can be detected with ultra violet detection (UVD), fluorescence detection $^{6}$ (FLD), and electrochemical detection (ECD) due to the two phenolic hydroxyl groups in its structure. Although UVD is commonly used as a detector of HPLC, ECD provides higher sensitivity than the other two types of detection methods

$\dagger$ To whom correspondence should be addressed.

E-mail: kenpc@ipc.kit.ac.jp and can be applied to practical fields such as the determination of BPA in serum. ${ }^{7,8}$

Even if we employed ECD, concentration of samples for trace analysis of BPA is still essential to achieve sensitivity at the ppt level; many factors affect the determination results in such a low concentration range. A column switching HPLC is one of the solutions for those difficulties. It can provide both low

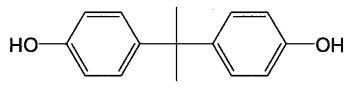

A

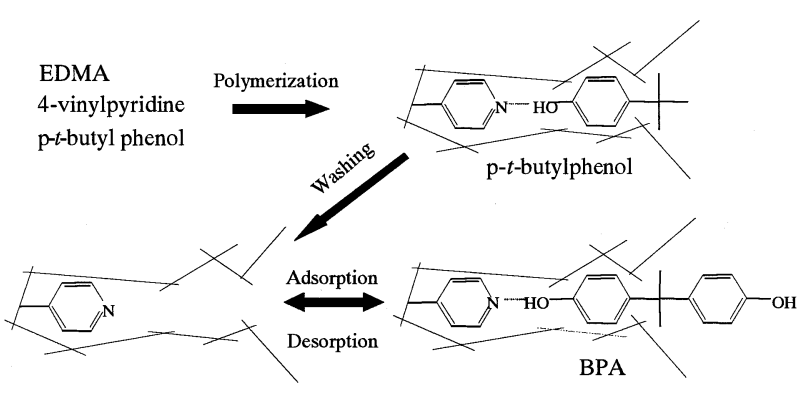
B

Fig. 1 Structure of BPA (A) and diagram of the imprinted site (B). 
contamination level and high efficiency of concentration. As a pretreatment column, we employed molecularly imprinted polymer (MIP). It was reported that MIPs were effective to remove interference during pretreatment processes..$^{9-12}$ The employed MIP was prepared with ethylene glycol dimethacrylate (EDMA) as a cross-linking agent, p-tertbutylphenol as a pseudo-template, and 4-vinylpyridine (4-VP) as a functional monomer. A diagram of the imprinted site is illustrated in Fig. 1-B.

For the reference, we performed BPA concentration manually and compared the results to those obtained by column switching auto-concentrating HPLC. In addition, we investigated methods for effectively preserving environmental water containing BPA. Long-term storage of environmental water is often accompanied by a reduction in levels of BPA, critically affecting samples with trace levels of the compound. Then, column switching auto-concentrating HPLC procedure was applied to actual determination of BPA in river and lake water samples.

\section{Experimental}

\section{Instrumentation and chemicals}

A lab-scale water purification system was purchased from Millipore (Bedford, MA, USA), which consisted of an Elix-UV and a Milli-Q Gradient. Provided water was purified through RO membrane (commercial membrane), ion exchange and UV lamp. Empore disk (47 mm o.d. of SDB-XD type consisting of styrene-divinylbenzene co-polymer), a membrane stationary phase, was purchased from 3M (St. Paul, MN, USA). Acetonitrile and methanol of HPLC grade, sodium phosphate dibasic, sodium dihydrogen phosphate, ethyl acetate, ethylenediaminetetraacetic acid (EDTA), and ascorbic acid were purchased from Wako Pure Chemicals (Osaka, Japan). The HPLC system including Shim-pack VP-ODS column (150 mm $\times 4.6 \mathrm{~mm}$ i.d., silica-based C18 stationary phase for analysis) was purchased from Shimadzu (Kyoto, Japan). The HPLC system consisted of a LC-10Avp solvent delivery pump, a LC6A sample concentration pump, a CTO-10Avp column oven, a SPD-10Avp UV detector, an FCV-12AH two-position flow changeover valve, an FCV-13AL six-port flow selection valve, an SIL-10ADvp automatic injector, an SCL-10A system controller and CLASS-VP work station software. A Coulochem $\Pi$ electrochemical detector (ECD) was purchased from ESA (Chelmsford, MA, USA).

HPLC mobile phase composition was $20 \mathrm{mM}$ (sodium) phosphate buffer $(\mathrm{pH} 7.0)$-acetonitrile $=60: 40(\mathrm{v} / \mathrm{v})$ for standard evaluation, whereas it was 65:35 (v/v) for actual sample determination (isocratic elution). Flow rates for analysis and pretreatment were 0.8 and $2.5 \mathrm{~mL} / \mathrm{min}$, respectively. The electrode potential for electrochemical detection was $+0.55 \mathrm{~V}$. The UV detection wavelength was $275 \mathrm{~nm}$; the column oven was operated at $40^{\circ} \mathrm{C}$. Purified water utilized to prepare a standard solution of BPA was filtrated with the Empore disk to remove the trace amounts of BPA prior to use.

All the pieces of glassware except volumetric apparatus were treated with heat at $400^{\circ} \mathrm{C}$.

Preparation of the uniformly-sized, molecularly imprinted polymer

The procedures for two-step swelling, polymerization, and after-treatment were performed as described in our previous work. ${ }^{13}$ Feed ratio was as follows: EDMA, $3 \mathrm{~mL}$; 4-VP, 0.34 $\mathrm{mL}$; toluene, $3 \mathrm{~mL}$; p-tert-butylphenol, $0.06 \mathrm{~g}$ : AIBN, $0.06 \mathrm{~g}$ (EDMA-4-VP-p-tert-butylphenol, 40:8:1 in mole ratio). The

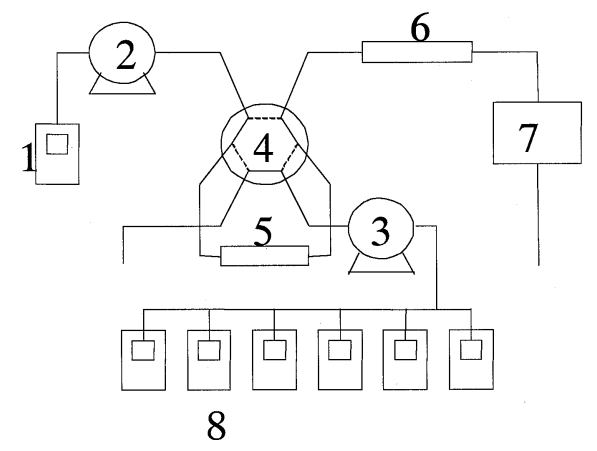

Fig. 2 Flow diagram of a column switching HPLC system: (1) mobile phase; $(2,3)$ pumps; (4) high pressure flow changeover valve; (5) pretreatment column; (6) analytical column; (7) ECD; (8) samples (one for rinsing solvent).

polymer particles obtained were $8 \mu \mathrm{m}$ in diameter. The size uniformity of the polymer particles was excellent, as reported previously. ${ }^{14}$ This polymer was packed into a stainless-steel column sized in $30 \mathrm{~mm} \times 4 \mathrm{~mm}$ i.d., using a slurry method. The column was utilized as a pretreatment device in column switching HPLC for determination of trace amounts of BPA.

\section{Membrane concentration of samples}

The Empore disk was set into the dedicated apparatus that consisted of a vacuum pump with a glass-attachment, a glassconcentrating tube, and a stainless-steel manifold with a threeway cock. $200 \mathrm{~mL}$ of BPA solutions or environmental water samples were treated. After extraction, the membrane dried of excess aspiration was washed with $2 \mathrm{~mL}$ of methanol and then 5 $\mathrm{mL}$ of ethyl acetate to elute the retained components. The extract was evaporated to dryness, followed by reconstitution with mobile phase for HPLC to make $1 \mathrm{~mL}$ of sample solution; then $50 \mu \mathrm{L}$ of it was injected onto HPLC by an automatic injector.

Carefully prepared 5 ppt and 20 ppt standard BPA solutions were analyzed five times each. Recovery and repeatability were evaluated. Based on the analysis of BPA free water, the BPA contamination from manual procedure was also estimated.

\section{Column switching HPLC}

To determine the BPA in water sample with complete suppression of BPA contamination, one requires a special technique. A column switching HPLC with pump injection system is one of the solutions. Figure 2 shows a flow diagram of the column switching HPLC system used in this study. The pump delivered $50 \mathrm{~mL}$ of BPA standard solution or environmental water sample to concentrate BPA on the pretreatment column packed with MIP. The pump-injection method provides rather more efficient concentration compared to fixed loop-injection with manual or automatic injector. Then mobile phase was delivered via six-port flow changeover valve; the BPA concentrated on the column was led to the analytical column and detected by ECD after the separation.

Carefully prepared 1, 10, and 100 ppt of standard BPA solutions were injected five times each and a calibration curve was created. Based on the calibration curve and repeatability, the detection limit of BPA was estimated statistically. Then the column switching HPLC was applied to actual determination of BPA. 


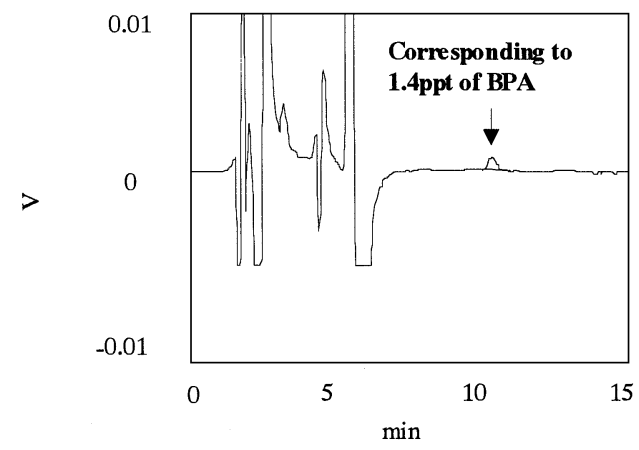

A

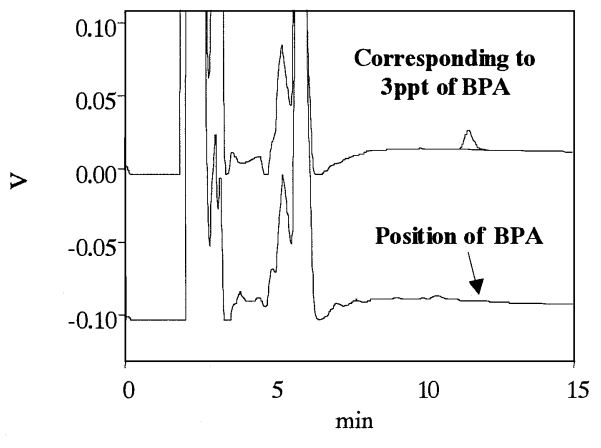

B

Fig. 3 BPA contaminations from manual pretreatment and purified water. A: Chromatogram of BPA contamination from manual membrane extraction. $200 \mathrm{~mL}$ of BPA free water was prepared, then injected as described in the experimental section. HPLC conditions: mobile phase, $20 \mathrm{mM}$ ( sodium) phosphate buffer $(\mathrm{pH}=7.0)$-acetonitrle $=60: 40(\mathrm{v} / \mathrm{v})$; flow rate, $0.8 \mathrm{~mL} / \mathrm{min}$; column, Shim-pack VP-ODS $(150 \mathrm{~mm} \mathrm{~L} \times 4.6 \mathrm{~mm}$ i.d.); detection, electro-chemical detector at $+0.55 \mathrm{~V}$; temperature, $40^{\circ} \mathrm{C}$; injection volume, $50 \mu \mathrm{L}$. B: Comparative chromatograms of purified water (upper) and of its filtrate (lower). Purified water was obtained from Milli-Q water purification system. The samples were analyzed with HPLC column switching system. Flow rate for preparation was $2.5 \mathrm{~mL} / \mathrm{min}$ and injection volume is $50 \mathrm{~mL}$, different from that of Fig. 3-A. HPLC conditions were the same as those in A.

\section{Storage of environmental water}

BPA was added into pond water to make $100 \mathrm{ppt}$. This water showed very little contamination. It was then kept during $15 \mathrm{~h}$ in glass and stainless-steel reservoirs containing $10 \mathrm{mM}$ of EDTA or ascorbic acid, then recoveries were evaluated with HPLC.

\section{Results and Discussion}

\section{Membrane concentration}

The concentrating process of BPA in environmental water samples requires a large volume to be treated due to its low concentration. Suspended solids are often contained in the samples, consequently the Empore disk having a large cross section area is quite suitable pretreatment media for manual pretreatment to avoid clogging.

A major benefit of manual pretreatment is the simple experimental apparatus. On the other hand, the drawback is poor reliability especially in the low concentration range. Manual pretreatment requires multi-step operations such as evaporation, filtration, re-dissolving, and so on. During these steps, BPA contamination might come from experimental environment. BPA contamination during pretreatment was measurably serious, therefore, we had to wash all the pretreatment devices with acetone or methanol carefully and treat all the glassware except volumetric apparatus at $400^{\circ} \mathrm{C}$ prior to use. For preventing the contamination, heating glassware higher than the boiling point of BPA $\left(250^{\circ} \mathrm{C}\right)$ before use might be effective. In the manual pretreatment of biological samples for BPA determination with GCMS, heating glassware before use reduced BPA contamination to one-third of that obtained with non-heat treated glassware.

For injection of sample solution, an automatic injector was superior to a manual injector. The reason is not only the repeatability of injection volume but also the degree of BPA contamination. In general, manual syringes with cemented needle provide unavoidable BPA contamination in microanalysis. ${ }^{15}$ This might be due to the adhesive used to fix each needle to the syringe body. Manual syringes with removable needle showed rather small BPA contamination in our preliminary investigation, but in this study we use automatic injector even in manual pretreatment to compare the manual and the column switching pretreatments correctly.

Through these pretreatment procedures, the overall BPA contamination was higher than $1.4 \mathrm{ppt}$ (Fig. 3-A). This was obtained by treating filtered pure water with no BPA contamination, as is described in a later paragraph.

The results of repeatability and recovery for 5 and $20 \mathrm{ppt}$ of BPA solutions are shown in Table 1. Based on these RSD values, 5 ppt of BPA seems to be determined, but the result of pond water to which $10 \mathrm{ppt}$ of BPA had been added showed a worse value, as $40 \%$ RSD. This might suggest the actual quantification limit of this method would be higher than $20 \mathrm{ppt}$. BPA contamination varied from one pretreatment to another; then the detection limit seemed rather higher than that of column switching method even if $1.4 \mathrm{ppt}$ could be detected. These results show that membrane pretreatment is not suitable for BPA determination when an expected concentration is below 10 ppt. The manipulative skill of the operator is important to minimize this contamination.

\section{Column switching HPLC}

A column switching HPLC has two major benefits for microanalysis. One is automated pretreatment and the other is automated sample concentration. If one uses this system, some manual procedures such as evaporation or transferences from one set of glassware to other pieces are not necessary. Then the loss of actual sample can be minimized. ${ }^{16}$ When the sample volume is large enough, on-column concentration is possible. ${ }^{17}$ Proper combination of pretreatment column and sample solvent can provide more than 1000 -fold concentration of sample for analysis. ${ }^{18}$

Using column switching HPLC method, the pump delivers the sample solution. Consequently, accuracy and precision of injection volume are dependent on flow rate and time program for pumps and switching valves. Thus, sample delivery pump was operated earlier and the starting point of pretreatment was 
Table 1 Recovery and repeatability of membrane concentration for standard BPA

\begin{tabular}{rcc}
\hline & Recovery, \% & Repeatability, \%RSD \\
\hline 20 ppt standard solution & 80 & 3.9 \\
5 ppt standard solution & 103 & 9.9 \\
\hline
\end{tabular}

Table 2 Recovery (\%) of BPA after overnight storage

\begin{tabular}{lccc}
\hline \multicolumn{1}{c}{ Additive } & Non & 10 mM EDTA & 10 mM Ascorbic acid \\
\hline Glass reservoir & 77.1 & 91.2 & 60 \\
Stainless-steel reservoir & 41.9 & 93.6 & 46.8 \\
\hline
\end{tabular}

programmed to change the position of two-position flow changeover valve automatically. This method provided rather sophisticated results.

Standard aqueous solutions of BPA at 1, 10, and 100 ppt were injected repeatedly $(n=5)$ to estimate the repeatability; the results were $9.3,3.4$ and $0.5 \%$ of $\mathrm{RSD}$, respectively. In addition, a linear calibration curve with a correlation coefficient greater than 0.999 was obtained. The recovery at $100 \mathrm{ppt}$ was $100.5 \%$ and not even a trace of leaking and/or carry-over of BPA was observed at the same concentration. The detection limit estimated based on standard deviation $(\sigma)$ of $y$-intercept of calibration curves was $0.36 \mathrm{ppt}$, which was obtained as follows: [3.3 $\sigma$ of $y$-intercept)/(slope value of calibration curve)]

The equation of the calibration curve was $y=78665 x-47475$, where $x$ is the concentration of BPA and $y$ is the peak area; $\sigma$ of the $y$-intercept was estimated based on the residual sum of squares. Here it was 8622; then the detection limit was calculated as $0.36 \mathrm{ppt}$.

BPA-free water can be obtained by filtrating purified water with the Empore disk, SDB-XD or C18 type. Both disks show strong BPA retention if applied with just water as the solvent. Then the filtrate can be used as BPA-free water for creating the calibration curve. Figure 3-B shows a comparison of the chromatogram of purified water and that of filtrated water. By this filtration with the Empore disk, BPA contamination was suppressed below the detection limit.

\section{Storage of environmental water}

It is commonly known that BPA concentrations of water samples from rivers or lakes gradually decrease as time goes on. Consequently, the same sample will give different quantitative results from day to day. Chemical reactions such as degradation or adsorption might occur. Yet this phenomenon was not observed when standard BPA solution prepared with purified water was stored for a long time. This suggests that some substances co-existing in environmental water are directly connected to the phenomenon. To find out proper precautionary measures, we evaluated the materials of sample reservoir containers and additives.

BPA was added to environmental water, which has very little BPA contamination, to make $100 \mathrm{ppt}$ of concentration and the mixture was allowed to stand for $15 \mathrm{~h}$ in different conditions as shown in Table 2. Then membrane extraction was performed to $200 \mathrm{~mL}$ of each water sample to compare the recovery.

The obtained results are shown in Table 2. Addition of EDTA promoted good recoveries of higher than $90 \%$ for both glass and stainless-steel reservoirs, whereas adding ascorbic acid provided poor recoveries for both reservoir materials. When we added
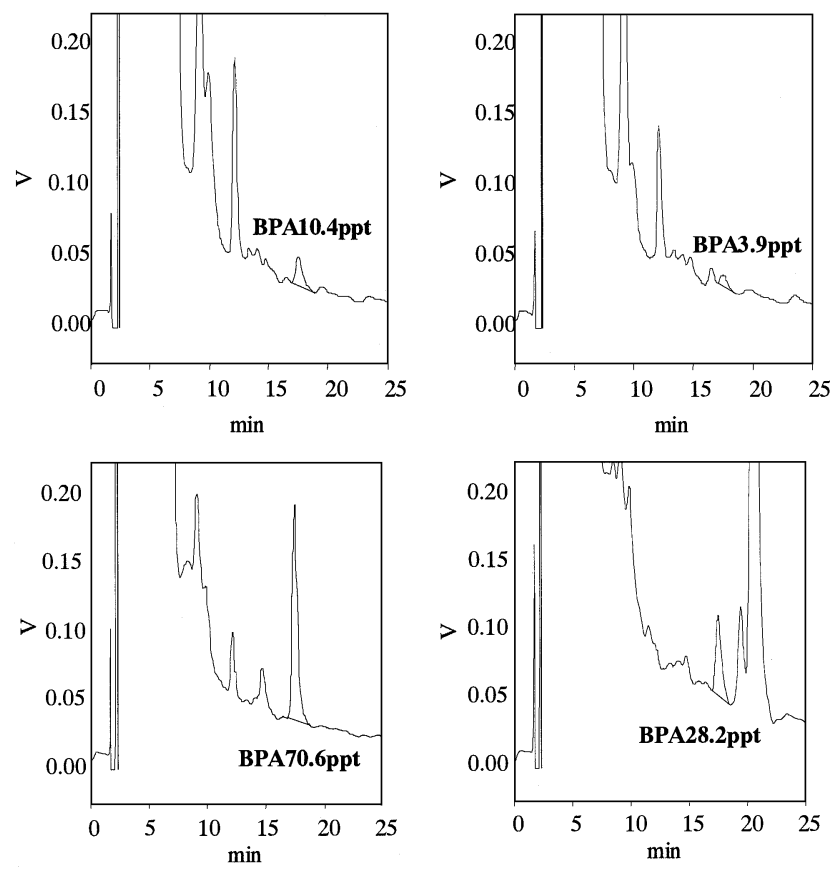

Fig. 4 Chromatograms of lake water samples obtained with column switching HPLC. $50 \mathrm{~mL}$ of lake water was concentrated onto a pretreatment column packed with molecularly imprinted polymer. HPLC conditions: mobile phase, $20 \mathrm{mM}$ (sodium); phosphate buffer $(\mathrm{pH} 7.0)$-acetonitrile $=65: 35(\mathrm{v} / \mathrm{v})$; flow rate, $0.8 \mathrm{~mL} / \mathrm{min}$; column, Shim-pack VP-ODS (150 mm L $\times 4.6 \mathrm{~mm}$ i.d.); detection, electrochemical detector at $+0.55 \mathrm{~V}$; temperature, $40^{\circ} \mathrm{C}$.

EDTA into environmental water samples to make $10 \mathrm{mM}$ of concentration during the sampling process, the fluctuations of recovery were suppressed. The two facts that the addition of ascorbic acid gave a negative result and that the decrease in BPA concentration could not be observed when we dissolved BPA into purified water, would suggest that this phenomenon was due to microbes in environmental water, not to some chemical reaction such as oxidation. EDTA has bactericidal activity. In this study, the recovery with glass reservoir was superior to that with stainless-steel reservoir. On the other hand, when BPA in tap water was investigated, the stainlesssteel reservoir provided better recovery than that of glass reservoir. This would be due to some co-existing substances in environmental water.

\section{Application to environmental water samples}

Based on the above mentioned results, actual determinations of water samples from Lake Kasumigaura and its branch rivers were preformed. Resulting chromatograms are shown in Fig. 4. Low concentrations of BPA were resolved without serious peak overlapping. In fact, the level of 1 ppt can be easily determined using this method. The repeatability $(n=5)$ of actual environmental water samples containing approximately $20 \mathrm{ppt}$ was $2.4 \%$ RSD. For the reference, a commercially available pretreatment column, Shimadzu SPC-RP3, was also applied to different river water analyses preliminarily; it gave a rather worse removal of interference, as is shown in Fig. 5.

\section{Conclusion}

It has been confirmed that BPA, which is often present in environmental water and has been brought to public attention as 


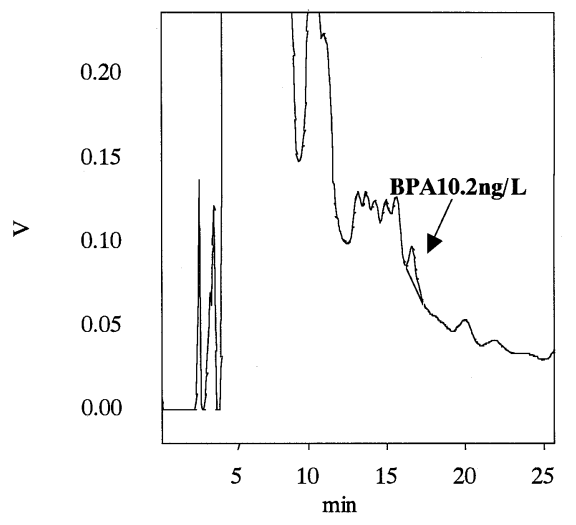

Fig. 5 Chromatograms of river water obtained with column switching HPLC coupled with SPC-RP3 pretreatment column. Pretreatment column: Shimadzu, $30 \mathrm{~mm} \mathrm{~L} \times 4 \mathrm{~mm}$ i.d., polyvinylalcohol resin, $9 \mu \mathrm{m}$. HPLC conditions were the same as in Fig. 4.

an endocrine disrupter, can be detected below 1 ppt level of concentration with column switching HPLC. Recent studies have revealed that BPA affects biological systems even at ultralow concentrations such as low ppt level. Consequently environmental studies to determine BPA at such low concentration levels will become even more important. Although the GCMS method provides good selectivity, its throughput and cost-performance are still drawbacks. On the other hand, HPLC column switching method provides simple pretreatment, good cost-performance and highly reliable results. The HPLC system provided background BPA contamination less than the detection limit. The most suitable HPLC method as demonstrated in this extensive study is the on-line HPLC column switching system and method. BPA below 1 ppt can be accurately and reproducibly detected using this HPLC system. Hopefully this methodology and HPLC system should provide a useful tool for more careful, accurate investigation and determination of BPA levels in the full spectrum of samples ranging from environmental to food products and consequently promote research on endocrine disruptors in biological matrices.

\section{Acknowledgements}

We gratefully acknowledge Professor K. Kaya, Tohoku University, for his helpful discussion. This research was partly supported by Nanotechnology Project of the Ministry of
Environment and Grant-in-Aid for Scientific Research (No 13640604 \& 14042232) from the Ministry of Education, Science, Sport, and Culture of Japan. In addition, financial support of this work by Shimadzu Science Foundation and Hosokawa Foundation is gratefully acknowledged.

\section{References}

1. L. H. Keith, "Environmental Endocrine Disruptors", 1997 Wiley, New York.

2. J. A. Brotons, M. F. Olea-Serrano, M. Villalobos, V. Pedraza, and N. Olea, Environ. Health Prespect., 1995 103, 608 .

3. F. S. von Saal, P. S. Cooke, D. L. Buchanan, P. Paianza, K. A. Thayer, S. C. Nagel, S. Parmigiani, and W. V. Welshons, Toxicol. Ind. Health, 1998, 14, 239.

4. J. S. Fisher, K. J. Turner, D. Brown, and R. M. Sharpe, Environ. Health Perpect., 1999, 107, 397.

5. R. Steinmetz, N. A. Mitchner, A. Grant, D. L. Allen, R. M. Bigsby, and N. Ben-Jonathan, Endocrinology, 1997, 138, 1780.

6. J. Haginaka, H. Sanbe, and K. Hosoya, Anal. Sci., 2003, 19, 715.

7. J. Sajiki, K. Takahashi, and J. Yonekubo, J. Chromatogr. $B, \mathbf{1 9 9 9}, 736,255$.

8. J. Sajiki, J. Chromatogr. B, 2001, 775, 9.

9. M. Quaglia, K. Chenon, A. J. Hall, E. D. Lorenzi, and B. Sellergren, J. Am. Chem. Soc., 2001, 123, 2146.

10. J. Matsuki, K. Fujiwara, and T. Takeuchi, Anal. Chem., 2000, 72, 1810

11. J. Haginaka and H. Sanbe, Anal. Chem., 2000, 72, 5206.

12. J. Jodlbauer, N. M. Maier, and W. Lindner, J. Chromatogr. A, 2002, 945, 45 .

13. R. A. Bartsch and M. Maeda, "Molecular and Ionic Recognition with Imprinted Polymers", 1998, American Chemical Society, Washington, D.C.

14. K. Hosoya and J. M. J. Frechet, J. Polym. Sci., Part A Polym. Chem., 1993, 31, 2129.

15. Y. Watabe, T. Kondo, H. Imai, M. Morita, N. Tanaka, and K. Hosoya, Anal. Chem., in press.

16. A. K. Sakhi, T. E. Gundersen, S. M. Ulven, R. Blomhoff, and E. Lundanes, J. Chromatogr. A, 1998, 828, 451.

17. H. S. Lee, C. K. Jeong, H. M. Lee, S. J. Choi, K. S. Do, K Kim, and Y. H. Kim, J. Chromatogr. A, 1999, 848, 179.

18. T. Kubo, K. Hosoya, Y. Watabe, T. Ikegami, N. Tanaka, T. Sano, and K. Kaya, J. Chromatogr. A, 2003, 987, 389. 